\title{
Checklist dos percevejos-do-mato (Hemiptera: Heteroptera: Pentatomoidea) do Estado de São Paulo, Brasil
}

\author{
Jocélia Grazia ${ }^{1,3}$ \& Cristiano Feldens Schwertner ${ }^{2}$ \\ ${ }^{1}$ Programa de Pós-graduação em Biologia Animal, Departamento de Zoologia, Instituto de Biociências, \\ Universidade Federal do Rio Grande do Sul-UFRGS, Av. Bento Gonçalves, n. 9500, \\ Bloco IV, Prédio 43435, CEP 91501-970, Porto Alegre, RS, Brasil \\ ${ }^{2}$ Departamento de Ciências Biológicas, Universidade Federal de São Paulo - UNIFESP, Campus Diadema, \\ Rua Artur Riedel, n. 275, Eldorado, CEP 09972-270, Diadema, SP, Brasil, e-mail: schwertner@unifesp.br \\ ${ }^{3}$ Autor para correspondência: Jocélia Grazia, e-mail: jocelia@ufrgs.br
}

GRAZIA, J. \& SCHWERTNER, C.F. Checklist of stink bugs (Hemiptera: Heteroptera: Pentatomoidea) from São Paulo State, Brazil. Biota Neotrop. 11(1a): http://www.biotaneotropica.org.br/v11n1a/en/abstract?i nventory+bn0371101a2011.

\begin{abstract}
Pentatomoidea knowledge in São Paulo state is here updated. Two hundred and two species in 92 genera belonging to Acanthosomatidae, Canopidae, Cydnidae, Pentatomidae, Phloeidae, Scutelleridae, Tessaratomidae and Thyreocoridae are registered. Forty one species were added and 13 taxonomical and nomenclatorial corrections were made comparing with the data presented in the first edition of the Biota SP in 1999. Pentatomoidea species richness of São Paulo state is around $25 \%$ of the species known to Brazil and around $3 \%$ of the world fauna. The increase of the studies in scarcely known families in Brazil will certainly raise these numbers. Pentatomidae was the most numerous taxon in São Paulo state, with $80 \%$ of the registered species.
\end{abstract}

Keywords: stink bugs, pentatomids, biodiversity of the State of São Paulo, BIOTA/FAPESP Program.

Number of species: in the world: +7,000, in Brazil: +820, estimated in São Paulo State: 450 .

GRAZIA, J. \& SCHWERTNER, C.F. Checklist dos percevejos-do-mato (Hemiptera: Heteroptera: Pentatomoidea) do Estado de São Paulo, Brasil. Biota Neotrop. 11(1a): http://www.biotaneotropica.org.br/ v11n1a/pt/abstract?inventory+bn0371101a2011.

Resumo: O conhecimento de Pentatomoidea no Estado de São Paulo é aqui atualizado. São registradas 92 espécies em 89 gêneros pertencentes as famílias Acanthosomatidae, Canopidae, Cydnidae, Pentatomidae, Phloeidae, Scutelleridae, Tessaratomidae e Thyreocoridae. Comparando com os dados apresentados na primeira edição do Biota SP em 1999, foram adicionadas 41 espécies além de 13 correções nomenclaturais e taxonômicas efetuadas. O Estado de São Paulo apresenta uma riqueza total ao redor de $25 \%$ das espécies conhecidas para o Brasil e ao redor de $3 \%$ da fauna mundial de Pentatomoidea. Com o incremento dos estudos em famílias pouco conhecidas no país, estes números certamente serão ampliados. Pentatomidae resultou no táxon mais numeroso no estado, com $80 \%$ de espécies registradas.

Palavras-chave: percevejos-do-mato, pentatomídeos, biota paulista, Programa BIOTA/FAPESP.

Número de espécies: no mundo: +7.000, no Brasil: +820, estimadas no Estado de São Paulo: 450. 


\section{Introdução}

A superfamília Pentatomoidea inclui na sua maioria percevejos fitófagos. Os adultos podem ser reconhecidos pelo corpo geralmente ovalado, antenas com cinco segmentos e escutelo desenvolvido, sempre ultrapassando a metade do comprimento do abdome (Schuh \& Slater 1995, Grazia et al. 1999b). É o táxon mais diverso da infraordem Pentatomomorpha, tendo surgido muito cedo na evolução do grupo (Henry 1997). Compreende cerca de 7.000 espécies no mundo incluídas em 15 famílias (Grazia et al. 2008), das quais Acanthosomatidae, Canopidae, Cydnidae, Dinidoridae, Megarididae, Pentatomidae, Phloeidae, Scutelleridae, Tessaratomidae e Thyreocoridae são encontradas na região Neotropical (Grazia et al. 1999b no prelo, a). As sinapomorfias que suportam a monofilia do grupo incluem: escutelo ultrapassando a metade do comprimento do abdome, tricobótrios abdominais pareados e localizados lateralmente à linha dos espiráculos, abertura da cápsula genital dos machos (= pigóforo) direcionada posteriormente, ovos em forma de barril, ovóides ou esféricos (Henry 1997, Grazia et al. 2008). O monofiletismo dos táxons incluídos em Pentatomoidea bem como suas relações filogenéticas foram discutidas por Grazia et al. (2008).

Chaves e diagnoses para identificação das famílias e subfamílias de Pentatomoidea que ocorrem na região Neotropical são encontradas em Grazia et al. (no prelo a). Outras referências gerais importantes para o grupo no Brasil são Costa Lima (1940), Silva et al. (1968; revisada por Grazia 1977) e Grazia et al. (1999b).

\section{Metodologia}

A lista das espécies de Pentatomoidea registradas para o Estado de São Paulo (Tabela 2) foi elaborada com base na literatura (vide bibliografia) e na identificação de material. Grande parte dessas identificações é inédita e foram obtidas de material recebido de museus, coleções entomológicas e/ou pesquisadores; exemplares "voucher" encontram-se depositados na coleção do Departamento de Zoologia da Universidade Federal do Rio Grande do Sul (DZRS). Em relação à classificação de Pentatomidae, novas propostas em nível de tribo têm sido utilizadas (e.g. Cassis \& Gross 2002, Rider 2006), o que tem modificado consideravelmente a configuração dos grupos de gêneros, principalmente na subfamília Pentatominae. Neste novo contexto, os gêneros neotropicais anteriormente incluídos em Pentatomini (e.g. Grazia et al. 1999) estão organizados em oito tribos distintas, das quais sete ocorrem no estado de São Paulo. Essa proposta de classificação pode ser encontrada em Rider (2010b).

\section{Resultados e Discussão}

Para o Estado de São Paulo, são registradas para Pentatomoidea 201 espécies distribuídas em 90 gêneros, 9 tribos, 5 subfamílias e 8 famílias. Comparando com os dados apresentados em Grazia et al. (1999b), foram adicionadas 41 espécies e 13 correções taxonômicas e/ou nomenclaturais foram efetuadas. A diversidade dos pentatomóideos na região Neotropical, no Brasil e no Estado de São Paulo está representada na Tabela 1.

\section{Acanthosomatidae}

Tamanho médio (5 a $10 \mathrm{~mm}$ ), as espécies neotropicais têm coloração predominantemente verde ou castanha. No mundo, abrange cerca de 200 espécies e mais de 50 gêneros (Kumar 1974, Faúndez 2009). Inclui três subfamílias: Acanthosomatinae, Blaudinae (com duas tribos) e Ditomotarsinae (duas tribos). A distribuição é predominantemente austral, incluindo sul da África e da Argentina, Chile e Austrália; apenas a subfamília Acanthosomatinae tem espécies com distribuição nas regiões

Tabela 1. Número de gêneros e espécies das famílias de Pentatomoidea (Hemiptera) conhecidas ou estimadas para a região Neotropical, Brasil e para o Estado de São Paulo, incluíndo subfamílias de Pentatomidae ${ }^{1}$.

Table 1. Number of the known or estimated genera and species of Pentatomoidea(Hemiptera) families in the Neotropical region, Brazil and São Paulo state, including Pentatomidae subfamilies ${ }^{1}$.

\begin{tabular}{|c|c|c|c|c|c|c|}
\hline & Neotrópico & \multirow{2}{*}{ Espécies } & Brasil & \multirow{2}{*}{ Espécies } & São Paulo & \multirow{2}{*}{ Espécies } \\
\hline & Gêneros & & Gêneros & & Gêneros & \\
\hline Tessaratomidae & 1 & 3 & 1 & 2 & 1 & 1 \\
\hline Phloeidae & 2 & 3 & 2 & 3 & 2 & 3 \\
\hline Canopidae* & 1 & 8 & 1 & 6 & 1 & 2 \\
\hline Dinidoridae & 1 & 6 & 1 & 4 & 0 & 0 \\
\hline Pentatomidae & 228 & +1313 & +100 & +620 & 71 & 163 \\
\hline Asopinae & 21 & 91 & 14 & 46 & 8 & 13 \\
\hline Cyrtocorinae* & 4 & 11 & 4 & 9 & 2 & 3 \\
\hline Discocephalinae* & 75 & 278 & 31 & 124 & 14 & 25 \\
\hline Edessinae* & 5 & 280 & 4 & +100 & 4 & 16 \\
\hline Pentatominae & 122 & +600 & +70 & 338 & 43 & 105 \\
\hline Stirotarsinae* & 1 & 1 & 0 & 0 & 0 & 0 \\
\hline Scutelleridae & -- & -- & -- & +50 & 3 & 5 \\
\hline Cydnidae & 14 & 139 & 8 & +45 & 8 & 14 \\
\hline Thyrecoridae & 9 & 157 & 6 & 84 & 3 & 13 \\
\hline Acanthosomatidae & 18 & 22 & 1 & 1 & 1 & 1 \\
\hline Megarididae* & 1 & 16 & 1 & 6 & 0 & 0 \\
\hline TOTAL & +277 & +1667 & +120 & +820 & 92 & 202 \\
\hline
\end{tabular}

${ }^{1}$ Famílias, com exceção de Megarididae, arranjadas em ordem filogenética; subfamílias em ordem alfabética (conforme Grazia et al. 2008); (*) táxons com distribuição exclusivamente neotropical; (--) falta de dados para o táxon; and (+) indica que o número de táxons é superior.

${ }^{1}$ Families arranged in phylogenetic order, except Megarididae; subfamilies in alphabetic order (according to Grazia et al. 2008); (*) taxa exclusively neotropical; (--)missing data;(+)indicates a higher taxa number 
Neártica e Paleártica. A fauna mundial foi monografada por Kumar (1974). Chaves para os gêneros do hemisfério ocidental são encontradas em Rolston \& Kumar (1975). A única espécie até agora registrada em São Paulo foi Hellica nitida Haglund.

\section{Canopidae}

Percevejos de tamanho médio (5 a $8 \mathrm{~mm}$ ), totalmente negros e brilhantes, dorso fortemente convexo e escutelo amplamente desenvolvido, recobrindo todo o abdome e a maior parte dos hemiélitros. Distribuição exclusivamente neotropical, com um gênero (Canopus Fabricius) e oito espécies. McHugh (1994) reportou ninfas e adultos de diferentes espécies ocorrendo sobre fungos, determinando que esporos dos mesmos fungos estavam presentes no sistema digestivo destes insetos, confirmando assim seus hábitos micetófagos. Para São Paulo foram registradas duas espécies $C$. burmeisteri McAtee \& Malloch e $C$. orbicularis Horvath. Referências básicas, incluindo chave para identificação das espécies, são encontradas em McAtee \& Malloch (1928).

\section{Cydnidae}

Chamados vulgarmente de percevejos-cavadores, têm tamanho variável (3 a $10 \mathrm{~mm}$ ), predominantemente negros ou castanhos, geralmente brilhantes, cabeça alargada e achatada, pernas adaptadas para o hábito cavador. Tem distribuição mundial, estando bem representada nas regiões tropicais e temperadas, perfazendo mais de 750 espécies e 120 gêneros. Referência básica para a família na região Neotropical é a obra de Froeschner (1960), que inclui chaves para a identificação de famílias e espécies do novo mundo. Becker (1967) revisou as espécies neotropicais de Cephalocteinae, que inclui entre outras Scaptocoris castanea Perty e $S$. carvalhoi Becker, espécies que constituem sérias pragas em diferentes regiões do Brasil (Lis et al. 2000). A nomenclatura dessas espécies foi atualizada por Grazia et al. (2004); mais recentemente diversos trabalhos visando maior conhecimento sobre biologia e manejo dessas espécies têm sido feitos (i.e. Oliveira \& Malaguido 2004, Marques et al. 2005, Xavier \& Avila 2006, Čokl et al. 2006, Nardi et al. 2007, 2008). A taxonomia do grupo na região Neotropical está desatualizada. Quatorze espécies desta família foram registradas para São Paulo, distribuídas nas subfamílias Cephalocteinae e Cydninae.

Dinidoridae

Apesar de seu tamanho relativamente grande $(10$ a $30 \mathrm{~mm})$ e sua coloração aposemática, estes percevejos raramente estão representados em coleções. Compreende 115 espécies em 13 gêneros, sendo predominantemente encontrados nas regiões Afrotropical e Oriental. A família foi revisada por Durai (1987), que reconheceu as subfamílias Dinidorinae (com duas tribos) e Megymeninae (duas tribos); Kocorek \& Lis (2000) propuseram uma nova tribo para Megymeninae. Chaves para identificação dos táxons podem ser encontradas em Durai (1987) e Kocorek \& Lis (2000). Rolston et al. (1996) sumarizaram a classificação corrente para a família; Schaefer et al. (2000) revisaram a biologia das espécies de importância econômica, nenhuma delas com ocorrência para o Brasil. O conhecimento da biologia das espécies neotropicais se restringe à dados de etiqueta, principalmente registros de plantas-hospedeiras. Dinidor mactabilis Perty foi registrada formando agrupamentos em Smilax japecanga Grisebach (Smilacaceae) no sul do Brasil (Schwertner \& Grazia no prelo). A ausência de registro de espécies de Dinidoridae para o Estado de São Paulo (Tabela 1) certamente é resultado da falta de coleta.

\section{Megarididae}

Tamanho diminuto (cerca de $5 \mathrm{~mm}$ ou menos) e forma coleopteróide. É exclusivamente neotropical, com 16 espécies em um único gênero, Megaris Stål. A única chave para identificação das espécies é encontrada em McAtee \& Malloch (1928). A biologia da família é praticamente desconhecida (Schwertner \& Grazia no prelo), mas assume-se que suas espécies sejam exclusivamente fitófagas. Existem registros de Megaris puertoricensis Barber e M. semiamicta McAtee \& Malloch em Eugenia spp. (Myrtaceae) (ver referências em Schuh \& Slater 1995). A ausência de registro para o estado de São Paulo (Tabela 1) também resulta de falta de coleta, como mencionado para Dinidoridae.

\section{Pentatomidae}

Corresponde à quarta família mais numerosa e diversa entre os heterópteros, estando bem representada nas principais regiões faunísticas. Inclui mais de 4.500 espécies em 760 gêneros no mundo (Grazia et al. no prelo, a). Tamanho médio a grande (5 a $20 \mathrm{~mm}$ ), várias espécies são relacionadas a plantas cultivadas, algumas delas são pragas de importantes culturas (Panizzi et al. 2000). Constitui um grupo monofilético, incluindo oito subfamílias (Grazia et al. 2008); dessas, estão representadas na região Neotropical as subfamílias Asopinae, Cyrtocorinae, Discocephalinae, Edessinae, Pentatominae e Stirotarsinae. Asopinae inclui o único grupo de Pentatomoidea com hábitos predadores, condição que evoluiu secundariamente. Cyrtocorinae, Discocephalinae, Edessinae e Stirotarsinae têm distribuição exclusiva no neotrópico. Todas as subfamílias, com exceção de Stirotarsinae, estão representadas no Estado de São Paulo. Para Discocephalinae, as duas tribos propostas por Rolston (1992) tem registros em São Paulo (Discocephalini - 18 spp. e Ochlerini - 7 spp.); das tribos propostas por Rider (2010b), para Pentatominae, sete estão representadas no estado de São Paulo, a saber Catacanthini (8 spp.), Carpocorini (45 spp.), Menidini (1 spp.), Nezarini (12 spp.), Pentatomini (32 spp.), Piezodorini (1 spp.) e Procleticini (1 spp.). Referências básicas para a família no hemisfério ocidental, com chaves para identificação de gêneros, são os trabalhos de Rolston et al. (1980) e Rolston \& McDonald (1981, 1984); McDonald (1981, 1984); e ainda Packauskas \& Schaefer (1998 - Cyrtocorinae) e Tomas (1992 Jurberg- Asopinae). Desde a década de 1960, Grazia e colaboradores vêm contribuindo com a descrição de novos táxons, revisões de gêneros e estudos de imaturos e biologia de pentatomídeos, incluindo espécies com registro para o Estado de São Paulo (i.e. Grazia 1967, 1968, 1978, 1997, Grazia et al. 1980, 1999a, Martins et al.1986, Vecchio \& Grazia 1993, Grazia 1997, Schwertner et al. 2002, Fortes \& Grazia 2005, Campos \& Grazia 2006, Fernandes \& Grazia 2006, Schwertner \& Grazia 2007, Bernardes et al. 2009, Matesco et al. 2009; Rider (2010a) para lista de todas as publicações de J. Grazia). A biologia do grupo foi sumarizada recentemente por Grazia \& Schwertner (2008).

Phloeidae

Família de percevejos com aspecto peculiar, em função da morfologia externa críptica. São achatados, com as margens da cabeça, tórax e segmentos abdominais expandidos em grandes lobos, que se confundem com o substrato das cascas das árvores onde vivem (Lent \& Jurberg 1965, Grazia et al. no prelo a). Espécies de tamanho grande (20 a $25 \mathrm{~mm})$, são também chamados bichos-casca (Salomão et al. dados não publicados). Compreende 4 espécies em 3 gêneros (Grazia et al. 2008), com distribuição disjunta no Hemisfério Sul: Serbana 
Distant, 1906 (1 espécie) ocorre somente em Bornéo (Leston 1953), enquanto as espécies dos gêneros Phloea Spinola, 1837 (2 espécies) e Phloeophana Leston, 1953 (1 espécie) ocorrem apenas na América do Sul e parecem ter sua distribuição restrita ao Brasil registradas desde o Pará até o Rio Grande do Sul, associadas às regiões da Floresta Amazônica e Mata Atlântica. Lent \& Jurberg (1965) fizeram a revisão e caracterização do grupo, indicando como plantas hospedeiras araçá (Psidium sp, Myrtaceae), amendoeira (Terminalia catappa L., Rosaceae), figueiras (Ficus spp., Moraceae), guaraiúva (Sucurinega guaraiva Kuhlmann, Euphorbiaceae), imbaúba (Cecropia sp., Urticaceae), jaboticabeira (Eugenia cauliflora de Berg, Myrtaceae), paricágrande (Parkia multijuga Benth., Mimosaceae), tamboril (Enterolobium maximum Ducke) e cambuí (Myrcia sp., Myrtaceae). Mais recentemente, Guilbert (2003) e Bernardes et al. (2005) estudaram aspectos da biologia e da morfologia dos imaturos de Phloea subquadrata Spinola. Salomão et al. (dados não publicados) sumarizam o conhecimento sobre os bichoscascas na Serra do Japi, São Paulo.

Scutelleridae

Reúne heterópteros das mais variadas cores (vermelho, azul, amarelo, ente outras), frequentemente iridescentes. São conhecidos vulgarmente por percevejos-escudo, em face de seu amplo escutelo recobrindo todo o abdome. De tamanho médio a grande ( 5 a15 mm), têm distribuição mundial, com 80 gêneros e 450 espécies. São facilmente confundidos com besouros. É pouco estudada na região neotropical, mesmo estando relativamente bem representada nas coleções dos museus. Uma das espécies mais comuns é Pachycoris torridus (Scopoli), que tem ampla distribuição na região neotropical e apresenta grande variabilidade de coloração (Monte 1937, Sanchez-Souto et al. 2004). Entre os escutelerídeos é comum a ocorrência de espécies que apresentam policromatismo (i.e. Paleari 1992, Sanches-Soto et al. 2004). A biologia das espécies de importância econômica foi revisada por Javahery et al. (2000), incluíndo entre elas pelo menos três espécies do gênero Pachycoris Burmeister (Javahery et al. 2000, Soto \& Nakano 2002). Para São Paulo foram registradas cinco espécies. A taxonomia e a biologia do grupo no Brasil e na região Neotropical está desatualizada. Barcellos et al. (no prelo) sumarizam as informações sobre a familia na Argentina fornecendo uma chave para os gêneros e a relação das plantas hospedeiras.

Tessaratomidae

Assemelham-se a grandes pentatomídeos (podem ultrapassar $40 \mathrm{~mm}$ ), dos quais se distinguem pela cabeça muito pequena em relação ao tamanho do corpo, antenas geralmente com quatro artículos, rostro curto, raramente ultrapassando as coxas anteriores e pronoto estendendo-se sobre a base do escutelo. Rolston et al. (1993) sumarizaram a classificação corrente para a família. São conhecidos 45 gêneros e cerca de 230 espécies com distribuição predominante nos trópicos do Velho Mundo. Inclui três subfamílias: Tessaratominae, Natalicolinae e Oncomerinae. Na região Neotropical, apenas o gênero cosmopolita Piezosternum Amyot \& Serville está representado, com três espécies. Schaefer et al. (2000) revisaram a biologia das espécies de importância econômica, nenhuma delas com ocorrência para o Brasil. O conhecimento sobre as espécies neotropicais se restringe a dados de etiqueta, principalmente registros de plantas-hospedeiras. Espécies de Piezosternum foram registradas em plantas das famílias Curcubitacea, Rubiaceae, Malvaceae, Myrtaceae e Solanaceae (Maes 1994, Schwertner
\& Grazia no prelo). Apenas Piezosternum thunbergi Stål foi registrada para São Paulo.

Thyreocoridae

De pequenos a médios ( 3 a $8 \mathrm{~mm}$ ), estes percevejos têm coloração escura, escutelo bem desenvolvido, convexo, recobrindo todo abdome e a maior parte da asa anterior; geralmente o exocório é amarelado. Tratados por alguns autores como subfamília de Cydnidae, os percevejos-negros, como são conhecidos vulgarmente, reúnem 213 espécies em 12 gêneros no mundo, sendo nove no hemisfério ocidental (subfamília Corimelaeninae) e três gêneros monotípicos no hemisfério oriental (subfamília Thyreocorinae) (Schuh \& Slater 1995, Lis 2006, Grazia et al., no prelo, b). Apesar de desatualizada, McAtee \& Malloch (1933) ainda é a única referência contendo chaves para gêneros, subgêneros e espécies. O gênero Galgupha Amyot \& Serville é o mais diverso, com 156 espécies em 15 subgêneros. São abundantes e bastante diversos, mas a biologia das espécies neotropicais é praticamente desconhecida (Grazia et al., no prelo, b). Treze espécies pertencentes a três gêneros foram registradas para São Paulo. A taxonomia do grupo na região Neotropical está desatualizada.

\section{Comentários sobre a lista, riqueza do estado comparado com outras regiões}

A riqueza total do grupo no Estado pode ser considerada alta, já que representa cerca de $25 \%$ das espécies conhecidas para o Brasil e cerca de $3 \%$ da fauna mundial. Se for levado em consideração famílias pouco coletadas e cuja taxonomia na região Neotropical necessita de urgente revisão (e.g. Cydnidae, Scutelleridae e Thyreocoridae), esse número certamente deve ser ampliado nos próximos anos. Aguiar et al. (2009) estimaram que para o Brasil ainda precisam ser descritos cerca de $20 \%$ da fauna de Hemiptera; para as famílias acima mencionadas esse percentual deve ser ainda maior, incluindo a fauna do estado de São Paulo.

A família mais numerosa no estado é Pentatomidae, que corresponde a $80 \%$ das espécies registradas. Esta é a família mais diversa de Pentatomoidea, sendo que em termos mundiais corresponde a $65 \%$ das espécies. Trabalhos recentes de levantamento de Pentatomoidea na região sul do Brasil (e.g. Schmidt \& Barcellos 2007, Campos et al. 2009, Mendonça Jr. et al. 2009) têm confirmado proporções mais altas, uma vez que várias famílias (e.g. Acanthosomatidae, Tessaratomidae) são bem menos diversas na região Neotropical em relação a outras regiões biogeográficas (ver Tabela 1 e textos introdutórios das respectivas famílias).

Para fins comparativos com outras regiões do neotrópico, poucos países têm listas de espécies para todas as famílias de Pentatomoidea, incluíndo Chile (Prado 2008), Colômbia (Grazia dados não publicados), Equador (Froeschner 1981), Guiana Francesa (Becker \& Grazia 1977), Haiti e República Dominicana (Perez-Gelabert 2005), Nicarágua (Maes 1994), Panamá (Froeschner 1999), Venezuela (Becker \& Grazia 1971) e Nordeste do Uruguai (Grazia \& Casini 1973). Para Honduras e México, as listas se restringem apenas à família Pentatomidae (Arismendi \& Thomas 2003, Thomas 2000). Para Argentina, listas de espécies das famílias Acanthosomatidae, Dinidoridae, Megarididae, Pentatomidae, Tessaratomidae e Thyreocoridae foram sintetizadas recentemente (Grazia \& Schwertner 2008, no prelo, Grazia et al. no prelo b, Schwertner \& Grazia no prelo). Em Grazia (1984) o conhecimento da tribo Pentatomini na Venezuela foi ampliado. Todos esses trabalhos apontam Pentatomidae como a família mais numerosa, variando de 87 espécies no Equador 
até 318 espécies no México. O bioma Pampa, que inclui o sul do Brasil, todo o território do Uruguai e o centro-leste da Argentina, apresenta cerca de 150 espécies de pentatomídeos (Grazia, Schwertner \& Simões, dados não publicados).

A fauna de percevejos-do-mato do Estado de São Paulo representa uma parcela importante da fauna neotropical, na qual estão presentes os principais táxons supragenéricos encontrados na região.

\section{Principais avanços relacionados ao Programa BIOTA/FAPESP}

Nestes dez anos que se passaram desde a publicação do trabalho de Grazia et al. (1999), vários novos táxons foram descritos incluindo material procedente de São Paulo, assim como novos registros (e.g. Fortes \& Grazia 2000, 2005, Frey-da-Silva \& Grazia 2001, Freyda-Silva et al. 2002, Campos et al. 2004, Silva et al. 2006). Como resultado, houve um acréscimo considerável no número de espécies, além das correções a atualizações nomenclaturais. Trabalhos sobre a filogenia e classificação (e.g. Barcellos \& Grazia 2003, Fortes \& Grazia 2005, Grazia et al. 2008), síntese (e.g. Grazia \& Schwertner 2008, Grazia et al. no prelo a, Schwertner \& Grazia no prelo) e levantamentos sobre táxons da superfamília foram ou estão sendo publicados, avançando consideravelmente o conhecimento desse grupo no Brasil, permitindo inclusive uma melhor estimativa de sua diversidade.

Cabe ressaltar a oportunidade da publicação do trabalho de Grazia et al. (1999) como um dos capítulos da obra Biodiversidade do Estado de São Paulo no volume sobre Invertebrados Terrestres (Brandão \& Cancello 1999). O capítulo sobre Pentatomoidea foi o primeiro trabalho de síntese feita sobre a biodiversidade do grupo no Brasil, em português, após mais de 50 anos, desde o trabalho de Costa Lima (1940). Dessa forma, se tornou referência básica para o grupo no nosso país, tendo papel importante na formação de recursos humanos que trabalham não só com percevejos mas com insetos em geral.

\section{Principais grupos de pesquisa em taxonomia}

Listamos a seguir os grupos de pesquisas que trabalham com taxonomia de Pentatomoidea no Brasil indicando as principais famílias em que atuam (Instituição e nome dos pesquisador(es) principal(ais):

- Universidade Federal do Rio Grande do Sul, Porto Alegre, RS. Jocelia Grazia (Pentatomoidea, Dinidoridae, Pentatomidae, Phloeidae, Thyreocoridae).

Luiz Alexandre Campos (Pentatomoidea, Pentatomidae: Discocephalinae, Pentatominae).

- Fundação Zoobotânica do Rio Grande do Sul, Porto Alegre, RS. Aline Barcellos (Scutelleridae, Pentatomidae) .

- Universidade Federal do Pará, Belém, PA.

José Antônio Fernandes (Pentatomidae: Discocephalinae, Edessinae, Pentatominae, Coreidae).

- Universidade Federal de São Paulo, Diadema, SP.

Cristiano Schwertner (Pentatomoidea, Pentatomidae, Cydnidae).

Nunca houve especialistas em taxonomia de nenhum grupo de Heteroptera trabalhando em Instituições do Estado de São Paulo até janeiro de 2009, quando ocorreu a contratação de C.F. Schwertner pela UNIFESP.

Outros grupos de pesquisa no Brasil ou no Estado de São Paulo trabalham em relação à agroecossistemas, principalmente com grupos de interesse econômico.

\section{Principais acervos}

A coleção mais significativa é sem dúvida a do Museu de Zoologia da USP (curador: Carlos Campaner). Outras coleções incluem acervos relativamente pequenos do Museu de Ciências da UNICAMP, Museu de Entomologia da UNESP \Ilha Solteira e aquelas centradas em plantas cultivadas, como as do Instituto Biológico (unidades de São Paulo, Campinas, Ribeirão Preto), Horto Florestal-IF\São Paulo, Instituto Agronômico de Campinas, ESALQ-USP\Piracicaba e UNESP \Jaboticabal.

No Brasil destacam-se ainda as coleções do Instituto Nacional de Pesquisas da Amazônia (INPA), Manaus, AM (Augusto Henriques), Museu de Ciências Naturais da Fundação Zoobotânica-RS, Porto Alegre, RS (Aline Barcellos), Museu Nacional-UFRJ, Rio de Janeiro, RJ (Jorge Nessimian, Luiz Costa) e Museu Paraense Emilio Goeldi, Belém, PA (Orlando Tobias da Silveira)

No Exterior, destacam-se as coleções do American Museum of Natural History, Nova Iorque, EUA (Randall T. Schuh), Museum National d'Histoire Naturelle, Paris, França (Eric Guilbert), National Museum of Natural History, Washington DC, EUA (Thomas J. Henry), Naturhistoriska Riksmuseet, Estocolmo, Suécia (Gunvi Lindberg), Nationaal Natuurhistoric Museum, Leiden, Holanda (Yvone van Nierop) e The Natural History Museum, Londres, Inglaterra (Mick Webb).

\section{Principais lacunas do conhecimento}

A representatividade da fauna de pentatomóideos do Estado de São Paulo em coleções no Brasil e no Exterior está associada à falta de coletas. Para esse grupo de percevejos, mas de maneira geral também para toda a subordem Heteroptera, um inventário sistemático ainda não foi realizado no Estado. Além de coletas de grupos de interesse econômico (em agroecossistemas) e outras coletas esporádicas feitas ao longo dos últimos três séculos, coletas mais regulares foram realizadas apenas recentemente, porém dentro de outros projetos de inventariamento (i.e. fauna de Hymenoptera e Diptera). Essas coletas podem ser consideradas eventuais, sendo que grande parte deste material ainda sequer foi estudado por algum dos grupos de pesquisa que trabalham com taxonomia de Pentatomoidea no Brasil. Como apontado por Grazia et al. (1999), ainda há necessidade de coletas no sul do Estado (em áreas de floresta) e no oeste (cerradões), mas de uma forma geral, coletas sistematizadas em todo estado certamente ampliariam o número de espécies registradas.

Além disso, as lacunas e diretrizes apontadas por Aguiar et al. (2009) para o estudo da ordem Hemíptera e demais grupos de insetos no Brasil também devem ser ressaltados para Pentatomoidea. Entre outros aspectos, vários táxons de pentatomóideos ainda não têm estudos cladísticos, fundamentais para classificação e ampliação do conhecimento desses grupos.

\section{Perspectivas de pesquisa com pentatomóideos para os próximos 10 anos}

A representatividade da fauna torna o estudo dos pentatomoídeos do Estado de São Paulo fundamental para a compreensão da evolução deste táxon na região Neotropical. Estudos de inventariamento devem ser considerados prioritários para o grupo, principalmente em relação às áreas indicadas por Grazia et al. (1999) e reforçadas acima. Além disso, deveriam ocorrer esforços no sentido de avançar no conhecimento de famílias que não receberam a ênfase necessária nos estudos da fauna de insetos no Estado de São Paulo até hoje, sejam eles taxonômicos, econômicos ou sob o ponto de vista da conservação da biodiversidade (i.e. Cydnidae, Dinidoridae, Megarididae, Thyreocoridae e as subfamílias Asopinae, Discocephalinae e Edessinae). 
É fundamental o apoio para estudos de sistemática (com ênfase na metodologia cladística) e o desenvolvimento de ferramentas de identificação e difusão dos conhecimentos sobre o grupo (e.g. chaves e manuais de identificação, banco de dados, etc.).

\section{Referências Bibliográficas}

AGUIAR, A.P., SANTOS, B.F., COURI, M.S., RAFAEL, J.A., COSTA, C., IDE, S., DUARTE, M., GRAZIA, J., SCHWERTNER, C.F., FREITAS, A.V.L. \& AZEVEDO, C.O. 2009. Insecta. In Estado da arte e perspectivas para a Zoologia no Brasil (R.M. Rocha \& W.A. Boeger, org.). Ed. UFPR, Curitiba, p.131-155.

ARISMENDI, N. \& THOMAS, D.B. 2003. Pentatomidae (Heteroptera) of Honduras: a checklist with description of a new ochlerine genus. Insecta Mundi 17(3-4):219-236.

BARCELLOS, A., EGER JUNIOR, J. \& GRAZIA, J. no prelo. Hemiptera: Scutelleridae. In Biodiversidad de Artrópodos Argentinos (S. Roig-Juñent, L.E. Claps \& J.J. Morrone, dir.) Tucumán, Sociedad Entomológica Argentina, v. 3

BARCELLOS, A. \& GRAZIA, J. 2003. Cladistic analysis and biogeography of Brachystethus Laporte (Heteroptera, Pentatomidae, Edessinae). Zootaxa 256:1-14.

BECKER, M. 1967. Estudos sobre a subfamília Scaptocorinae na região neotropical (Hemiptera: Cydnidae). Arq. Zool. S. Paulo 15:291-325.

BECKER, M. \& GRAZIA, J. 1971. Contribuição ao conhecimento da Superfamilia Pentatomoidea na Venezuela (Heteroptera). Iheringia, Sér. Zool. (40):3-26.

BECKER, M. \& GRAZIA. J. 1977. The Pentatomoidea (Heteroptera) collected in French Guyana by the expedition of the Muséum National d'Histoire Naturelle. Annls Soc. Ent. Fr. (N.S.) 13(1):53-67.

BERNARDES, J.L.C., GRAZIA J., BARCELLOS, A. \& SALOMÃO, A.T. 2005. Descrição dos estágios imaturos e notas sobre a biologia de Phloea subquadrata (Heteroptera, Phloeidae). Iheringia, Sér. Zool. 95(4):415-420.

BERNARDES, J.L.C., SCHWERTNER, C.F. \& GRAZIA, J. 2009. Cladistics analysis of Thoreyella and related genera (Hemiptera: Pentatomidae: Pentatominae: Procleticini). Zootaxa 2310:1-23.

BRANDÃO, C.R. \& CANCELLO, E.M. 1999. Biodiversidade do Estado de São Paulo, Brasil: síntese do conhecimento ao final do século XX. Invertebrados Terrestres. FAPESP, São Paulo, v.5.

CAMPOS, L.A., GRAZIA, J. \& GREVE, C. 2004. Notes on Catulona Rolston, 1992, and description of a new species from Brazil (Hemiptera, Pentatomidae, Discocephalinae). Zootaxa 404:1-7.

CAMPOS, L.A. \& GRAZIA, J. 2006. Análise cladística e biogeografia de Ochlerini (Heteroptera, Pentatomidae, Discocephalinae). Iheringia, Sér. Zool. 96(2):147-163.

CAMPOS, L.A., BERTOLINI, T.B.P., TEIXEIRA, R.A. \& MARTINS, F.S. 2009. Diversidade de Pentatomoidea (Hemiptera, Heteroptera) em três fragmentos de Mata Atlântica no sul de Santa Catarina. Iheringia, Sér. Zool. 99(2):165-171.

CASSIS, G. \& GROSS, G.F. 2002. Hemiptera: Heteroptera (Pentatomomorpha). In Zoological catalogue of Australia. (W.W.K. Houston \& A. Wells, ed.). CSIRO Publishing, Melbourne, Australia, v.27,3B.

C̆OKL, A., NARDI, C., BENTO, J.M.S., HIROSE, E. \& PANIZZI, A.R. 2006. Transmission of stridulatory signals of the burrower bugs, Scaptocoris castanea and Scaptocoris carvalhoi (Heteroptera: Cydnidae) through the soil and soybean. Physiol. Entomol. 31:371-381. http://dx.doi. org/10.1111/j.1365-3032.2006.00530.x

COSTA LIMA, A.M. 1940. Insetos do Brasil. Hemipteros. Escola Nacional de Agronomia, Rio de Janeiro, tomo2.

DURAI, P.S.S. 1987. A revision of the Dinidoridae of the world (Heteroptera: Pentatomoidea). Oriental Ins. 21:163-360.

FAÚNDEZ, E.I. 2009. Contribution to the knowledge of the genus Acrophyma Bergroth, 1917 (Hemiptera: Heteroptera: Acanthosomatidae). Zootaxa 2137:57-65.
FERNANDES, J.A.M. \& GRAZIA, J. 2006. Revisão do gênero Antiteuchus Dallas, 1851 (Heteroptera, Pentatomidae, Discocephalinae). Rev. Bras. Entomol. 50(1):165-231. http://dx.doi.org/10.1590/S008556262006000200004

FORTES, N.D.F. \& GRAZIA, J. 2000. Novas espécies de Rio Kirkaldy, 1909 (Heteroptera, Pentatomidae). Iheringia, Sér. Zool. (88):67-102.

FORTES, N.D.F. \& GRAZIA, J. 2005. Revisão e análise cladistica de Serdia Stal (Heteroptera, Pentatomidae, Pentatomini). Rev. Bras. Entomol. 49(3):294-339. http://dx.doi.org/10.1590/S0085-56262005000300002

FREY-DA-SILVA, A. \& GRAZIA, J. 2001. Novas espécies de Acrosternum subgênero Chinavia (Heteroptera, Pentatomidae, Pentatomini). Iheringia, Sér. Zool. (90):107-126.

FREY-DA-SILVA A., GRAZIA, J. \& FERNANDES, J.A.M. 2002. Revision of the genus Ogmocoris Mayr, 1864 (Heteroptera, Pentatomidae, Pentatomini). Beaufortia 52(10):179-185.

FROESCHNER, R.C. 1960. Cydnidae of the western hemisphere. Proc. U.S. Natl Mus. 111:337-680.

FROESCHNER, R.C. 1981. Heteroptera or true bugs of Ecuador: a partial catalog. Smithson. Contr. Zool. 322:1-147. http://dx.doi.org/10.5479/ si.00810282.322

FROESCHNER, R.C. 1999. True bugs (Heteroptera) of Panama: a synoptic catalog as a contribution to the study of Panamanian biodiversity. Memoirs of the American Entomological Institute, Washington.

GRAZIA, J. 1967. Estudos sobre o gênero Galedanta Amyot \& Serville, 1843 (Heteroptera, Pentatomidae). Iheringia, Sér. Zool. (35):45-59.

GRAZIA, J. 1968. Sobre o gênero Chloropepla Stal, 1867, com a descrição de uma nova espécie. (Hemiptera, Pentatomidae, Pentatomini). Rev. Bras. Biol. 28(2):193-206.

GRAZIA, J. 1977. Revisão dos pentatomídeos citados no "Quarto Catálogo dos Insetos que vivem nas Plantas do Brasil"'(Hemiptera, Pentatomidae, Pentatomini). Dusenia 10(3):161-174.

GRAZIA, J. 1978. Revisão do gênero Dichelops Spinola, 1837 (Heteroptera, Pentatomidae, Pentatomini). Iheringia, Sér. Zool. (53):1-119.

GRAZIA, J. 1984. Pentatomini da Venezuela (Heteroptera, Pentatomidae). An. Soc. Ent. Brasil 13(1):71-81.

GRAZIA, J. 1997. Cladistic analysis of the Evoplitus genus group of Pentatomini (Heteroptera: Pentatomidae). J. Comp. Biol. 2(1):43-48.

GRAZIA, J. \& CASINI, C.E. 1973. Lista preliminar dos heterópteros uruguaios da região nordeste: Pentatomidae e Coreidae. Iheringia, Sér. Zool. (44):55-63.

GRAZIA, J., CAVICHIOLI, R.R., WOLFF, V.R.S., FERNANDES, J.A.M. \& TAKIYA, D.A. no prelo a. Hemiptera. In Os insetos do Brasil: diversidade e taxonomia (J.A. Rafael, G.A.R. Melo, C.J.B. Carvalho \& S. Casari, org.).

GRAZIA, J., MATESCO, V.C. \& SCHWERTNER, C.F. no prelo b. Hemiptera: Thyreocoridae. In Biodiversidad de Artrópodos Argentinos (S. Roig-Juñent, L.E. Claps \& J.J. Morrone, dir.). Sociedad Entomológica Argentina, Tucumán, v.3.

GRAZIA, J., FERNANDES, J.A.M. \& SCHWERTNER, C.F. 1999a. Stysiana, a new genus and four new species of Pentatomini (Heteroptera: Pentatomidae) of the Neotropical region. Acta Soc. Zool. Bohem. 63(1-2):71-83.

GRAZIA, J., FORTES, N.D.F. \& CAMPOS, L.A. 1999b. Pentatomoidea. In Biodiversidade do estado de São Paulo, Brasil Heteroptera of economic importance (C.R. Brandão \& E.M. Cancello, ed.). FAPESP, São Paulo, p.421-474.

GRAZIA, J., SCHUH, R.T. \& WHEELER, W.C. 2008. Phylogenetic relationships of family groups in Pentatomoidea based on morphology and DNA sequences (Insecta: Heteroptera). Cladistics 24:932-976. http:// dx.doi.org/10.1111/j.1096-0031.2008.00224.x

GRAZIA, J. \& SCHWERTNER, C.F. 2008. Pentatomidae e Cyrtocoridae. In Biodiversidad de Artrópodos Argentinos (L.E. Claps, G. Debandi \& S. Roig-Juñent, dir.). Tucumán, Sociedad Entomologica Argentina, v.2, p.223-234. 
GRAZIA, J. \& SCHWERTNER, C.F. no prelo. Hemiptera: Acanthosomatidae In Biodiversidad de Artrópodos Argentinos (S. Roig-Juñent, L.E. Claps \& J.J. Morrone, dir.) Sociedad Entomológica Argentina, Tucumán, v.3.

GRAZIA, J., SCHWERTNER, C.F. \& SILVA, E.J.E. 2004. Arranjos taxonômicos e nomenclaturais em Scaptocorini (Hemiptera, Cydnidae, Cephalocteinae). Neotrop. Entomol. 33(4):511-512. http://dx.doi. org/10.1590/S1519-566X2004000400018

GRAZIA, J., VECCHIO, M.C., BALESTIERI, F.M.P. \& RAMIRO, Z.A 1980. Estudo das ninfas de pentatomídeos (Heteroptera) que vivem sobre soja (Glycine max (L.) Merrill): I - Euschistus heros (Fabricius, 1798) e Piezodorus guildinii (Westwood, 1837). An. Soc. Ent. Brasil 9(1):39-51.

GUILBERT, E. 2003. Habitat use and maternal care of Phloea subquadrata (Hemiptera: Phloeidae) in the Brasilian Atlantic forest (Espirito Santo). Eur. J. Entomol. 100:61-63.

HENRY, T.J. 1997. Phylogenetic analysis of family groups within the infraorder Pentatomomorpha (Hemiptera, Heteroptera), with emphasis on the Lygaeoidea. Ann. Entomol. Soc. Am. 90:275-301.

JAVAHERY, M., SCHAEFER, C.W. \& LATTIN, J.D. 2000. Shield bugs (Scutelleridae). In Heteroptera of economic importance (C.W. Schaefer \& A.R. Panizzi, ed.). CRC Press, Boca Ratón, p.505-512.

KOCOREK, A. \& LIS, J. 2000. A cladistic revision of the Megymeninae of the World (Hemiptera: Heteroptera: Dinidoridae). Pol. Pis. Entomol. 69(1):7-30.

KUMAR, R. 1974. A revision of world Acanthosomatidae (Heteroptera: Pentatomidae). Key to and description of subfamilies, tribes and genera, with designation of types. Aust. J. Zool. Supl. Series 34:1-60.

LENT, H. \& JURBERG, J. 1965. Contribução ao conhecimento dos Phloeidae Dallas, 1851, com um estudo sôbre genitália (Hemiptera, Pentatomoidea). Rev. Bras. Biol. 25:123-144

LESTON, D. 1953. "Phloeidae" Dallas: systematics and morphology, with remarks on the phylogeny of "Pentatomoidea" Leach and upon the position of "Serbana" Distant (Hemiptera). Rev. Bras. Biol. 13(2):121-140

LIS, J.A. 2006. Family Thyreocoridae Amyot \& Serville, 1843 - negro bugs. In Catalogue of the Heteroptera of the Palaeartic Region (B. Aukema \& C. Rieger, ed.). The Netherlands Entomological Society, Amsterdam, v.5, p.148-149.

LIS, J.A., BECKER, M. \& SCHAEFER, C.W. 2000. Burrower bugs (Cydnidae). In Heteroptera of economic importance (C.W. Schaefer \& A.R. Panizzi, ed.). CRC Press, Boca Ratón, p.505-512.

MAES, J.M. 1994. Catalogo de los Pentatomoidea (Heteroptera) da Nicaragua. Rev. Nicar. Entomol. 28:1-29.

MARQUES, O.M., AZEVEDO, R.L., LIMA JÚNIOR, C.A., CARVALHO, C.A.L. \& GIL-SANTANA, H.R. 2005. Scaptocoris carvalhoi, praga de pastagens na região do Recôncavo da Bahia. Bahia Agric. 7(1):73-74.

MARTINS, F.J.M., VECCHIO, M.C., GRAZIA, J. 1986. Estudo dos imaturos de Pentatomídeos (Heteroptera) que vivem sobre o arroz (Oriza sativa L.): I - Mormidea quinqueluteum (Lichtenstein, 1796). An. Soc. Ent. Brasil 15(2):349-359.

MATESCO, V.C., FÜRSTENAU, B.B.R.J., BERNARDES, J.L.C., SCHWERTNER, C.F. \& GRAZIA J. 2009. Morphological features of the eggs of Pentatomidae (Hemiptera: Heteroptera). Zootaxa 1984:1-30.

McATEE, W.L. \& MALLOCH, J.R. 1928. Sinopsis of pentatomid bugs of the subfamilies Megaridinae and Canopinae. Proc. U. S. Natl Mus. 72(25):1-21.

McATEE, W.L. \& J.R. MALLOCH. 1933. Revision of the subfamily Thyreocorinae of the Pentatomidae (Hemiptera-Heteroptera). Ann. Carnegie Mus. 21:191-411.

McHUGH, J.U. 1994. On the natural history of Canopidae (Heteroptera, Pentatomoidea). J. N. Y. Ent. Soc. 102:112-114

MENDONÇA JUNIOR, M.S., SCHWERTNER, C.F. \& GRAZIA, J. 2009. Diversity of Pentatomoidea (Hemiptera) in riparian forests of Bagé, RS, southern Brazil: taller forests, more bugs. Rev. Bras. Entomol 53(1):121-127. http://dx.doi.org/10.1590/S0085-56262009000100026

MONTE, O. 1937. Notas hemipterologicas. O Campo 8:70-72.
NARDI, C., FERNANDES, P.M., RODRIGUES, O.D. \& BENTO, J.M.S 2007. Flutuação populacional e distribuição vertical de Scaptocoris carvalhoi Becker (Hemiptera: Cydnidae) em área de pastagem. Neotrop. Entomol. 36:107-111. http://dx.doi.org/10.1590/S1519. 566X2007000100013

NARDI, C., FERNANDES, P.M. \& BENTO, J.M.S. 2008. Wing polymorphism and dispersal of Scaptocoris carvalhoi (Hemiptera: Cydnidae). An. Ent. Soc. Am. 101:551-557. http://dx.doi.org/10.1603/00138746(2008)101[551:WPADOS]2.0.CO;2

OLIVEIRA, L.J. \& MALAGUIDO, A.B. 2004. Flutuação e distribuição vertical da população do percevejo castanho da raiz, Scaptocoris castanea Perty (Hemiptera: Cydnidae), no perfil do solo em áreas produtoras de soja nas regiões centro-oeste e sudeste do Brasil. Neotrop. Entomol. 33:283-291.

PACKAUSKAS, R.J. \& SCHAEFER, C.W. 1998. Revision of the Cyrtocoridae (Hemiptera: Pentatomoidea). Ann. Ent. Soc. Am. 91:364-386.

PALEARI, L.M. 1992. Revisão do gênero Agonosoma Laporte, 1832 (Hemiptera, Scutelleridae). Rev. Bras. Entomol. 36:505-520.

PEREZ-GELABERT, D.E. \& THOMAS, D.B. 2005. Stink bugs (Heteroptera: Pentatomidae) of the island of Hispaniola, with seven new species from the Dominican Republic. Bol. Soc. Entomol. Aragonesa 37:319-352.

PANIZZI, R.A., McPHERSON, J.E., JAMES, D.G., JAVAHERY, M. \& McPHERSON, R.M. 2000. Stink bugs (Pentatomidae). In Heteroptera of economic importance (C.W. Schaefer \& A.R. Panizzi, eds.). CRC Press, Boca Raton, p.421-474.

PRADO, E. 2008. Conocimiento actual de Hemiptera-Heteroptera de Chile con lista de especies. Bol. Mus. Nac. Hist. Nat. 57:31-75.

RIDER, D.A. 2006. Family Tessaratomidae. In Catalogue of the Heteroptera of the Palaearctic Region. (B. Aukema \& C. Rieger, ed.). The Netherlands Entomological Society, Amsterdam, v.5, p.182-189.

RIDER, D.A. 2010a. Researchers: Jocélia Grazia. In Pentatomoidea Home page (D.A. Rider, org.). Fargo, North Dakota State University. Disponível em: http://www.ndsu.nodak.edu/ndsu/rider/Pentatomoidea/Researchers/ Grazia_Jocelia.htm (último acesso em 26/05/2010).

RIDER, D.A. 2010b. Classification. In Pentatomoidea Home page (D.A. Rider, org.). Fargo, North Dakota State University. Disponível em: http:// www.ndsu.nodak.edu/ndsu/rider/Pentatomoidea.htm (último acesso em 26/05/2010).

ROLSTON, L.H. 1992. Key and diagnoses for the genera of Ochlerini (Hemiptera: Pentatomidae: Discocephalinae. J. N. Y. Ent. Soc. 100(1):1-41.

ROLSTON, L.H. \& KUMAR, R. 1975 [1974]. Two new genera and two new species of Acanthosomatidae (Hemiptera) from South America, with a key to the genera of the Western Hemisphere. J. N. Y. Ent. Soc. 82(4):271-278.

ROLSTON, L.H., McDONALD, F.J.D. \& THOMAS JUNIOR, D.B. 1980. A conspectus of Pentatomini of the Western Hemisphere. Part 1. (Hemiptera: Pentatomidae). J. N. Y. Ent. Soc. 88(2):120-132.

ROLSTON, L.H. \& McDONALD, F.J.D. 1981. Conspectus of Pentatomini genera of the Western Hemisphere. Part 2. (Hemiptera: Pentatomidae). J. N. Y. Entomol. Soc. 88(4):257-282.

ROLSTON, L.H. \& McDONALD, F.J.D. 1984. A conspectus of Pentatomini of the Western Hemisphere. Part 3. (Hemiptera: Pentatomidae). J. N. Y. Entomol. Soc. 92(1):69-86.

ROLSTON, L.H., AALBU, R.L., MURRAY, M.J. \& RIDER, D.A. 1993. A catalogue of the Tessaratomidae of the World. Papua New Guinea Jl Agric. Forest. Fish. 36(2):36-108.

ROLSTON, L.H., RIDER, D.A., MURRAY, M.J. \& AALBU, R.L. 1996. Catalog of the Dinidoridae of the World. Papua New Guinea Jl Agric. Forest. Fish. 39(2):22-101.

SALOMÃO, A.T., POSTALI, T.C. \& VASCONCELLOS-NETO, J. dados não publicados. Bichos-cascas na Serra do Japi: história natural dos percevejos Phloeidae (Hemiptera). 
SANCHEZ-SOTO, S.S., MILANO, P. \& NAKANO, O. 2004. Nova planta hospedeira e novos padrões cromáticos de Pachycoris torridus (Scopoli) (Hemiptera: Scutelleridae) no Brasil. Neotrop. Entomol. 33:109-111. http://dx.doi.org/10.1590/S1519-566X2004000100019

SCHAEFER, C.W., PANIZZI, A.R. \& JAMES, D.G. 2000. Several small pentatomoid families (Cyrtocoridae, Dinidoridae, Urostylidae, Plataspidae, and Tessaratomidae). In Heteroptera of economic importance (C.W. Schaefer \& A.R. Panizzi, ed.). CRC Press, Boca Ratón, p.505-512. http://dx.doi.org/10.1201/9781420041859

SCHMIDT, L.S. \& BARCELLOS, A. 2007. Abundância e riqueza de espécies de Heteroptera (Hemiptera) do Parque Estadual do Turvo, sul do Brasil: Pentatomoidea. Iheringia, Sér. Zool. 97(1):73-79. http://dx.doi. org/10.1590/S0085-56262007000400005

SCHUH, T.R. \& SLATER, J.C. 1995. True bugs of the world (Hemiptera: Heteroptera): Classification and Natural History. Cornell University Press, Ithaca.

SCHWERTNER, C.F. \& GRAZIA, J. 2007. O gênero Chinavia Orian (Hemiptera, Pentatomidae, Pentatominae) no Brasil, com chave pictórica para os adultos. Rev. Bras. Entomol. 51(4):416-435.

SCHWERTNER, C.F. \& GRAZIA, J. no prelo. Hemiptera: Dinidoridae, Megarididae y Tessaratomidae. In Biodiversidad de artrópodes argentinos (S. Roig-Juñent, L.E. Claps \& J.J. Morrone, dir.). Sociedad Entomologica Argentina, Tucumán, v.3.

SCHWERTNER, C.F., ALBUQUERQUE, G.S. \& GRAZIA, J. 2002. Descriptions of the immature stages of Acrosternum (Chinavia) ubicum Rolston (Heteroptera: Pentatomidae) and effect of the host plant on size and coloration of nymphs. Neotrop. Entomol. 31(4):571-579. http:// dx.doi.org/10.1590/S1519-566X2002000400009
SILVA, A.G.A., GONÇALVES, C.R., GALVÃO, D.M., GONÇALVES, A.J.L., GOMES, J., SILVA, M.N. \& SIMONI, M.L. 1968. Quarto catálogo dos insetos que vivem nas plantas do Brasil, seus parasitos e predadores. Ministério da Agricultura, Rio de Janeiro.

SILVA, E.J.E.E., FERNANDES, J.A.M. \& GRAZIA, J. 2006. Caracterização do grupo Edessa rufomarginata e descrição de sete novas espécies (Heteroptera, Pentatomidae, Edessinae). Iheringia, Ser. Zoologia 96(3):345362.

SOTO, S.S. \& NAKANO, O. 2002. Ocorrência de Pachycoris torridus (Scopoli) (Hemiptera: Scutelleridae) em Acerola (Malpighia glabra L.) no Brasil. Neotrop. Entomol. 31:481-482. http://dx.doi.org/10.1590/ S1519-566X2002000300022

THOMAS JUNIOR, D.B. 1992. Taxonomic synopsis of the asopine Pentatomidae (Heteroptera) of the Western Hemisphere. Thomas Say Foundation Monograph, ESA, v.16.

THOMAS, D.B. 2000. Pentatomidae (Hemiptera). In Biodiversidad, Taxonomia y Biogeografia de artropodos de Mexico: hacia un sintesis de su conocimiento (J.E. Llorente, E. Gonzalez \& N. Papavero, ed.). Universidad Nacional Autonoma de Mexico, Mexico D.F., v.2., p.335-352.

VECCHIO, M.C. \& GRAZIA, J. 1993. Estudo dos imaturos de Oebalus ypsilongriseus (De Geer, 1773): III - Duração e mortalidade dos estágios de ovo e ninfa (Heteroptera: Pentatomidae). An. Soc. Entomol. Brasil 22(1):121-129.

XAVIER, L.M.S. \& ÁVILA, C.J. 2006. Patogenicidade de isolados de Metarhizium anisopliae (Metsch.) Sorokin e de Beauveria bassiana (Bals.) Vuillemin a Scaptocoris carvalhoi Becker (Hemiptera, Cydnidae). Rev. Bras. Entomol. 50(4):540-546. http://dx.doi.org/10.1590/S008556262006000400016 


\section{Apêndice}

Apêndice 1. Lista das espécies do Estado de São Paulo.

Appendix 1. List of the species of São Paulo state.

Os números apresentados junto aos nomes dos táxons representam respectivamente o número de espécies registradas para o Estado de São Paulo e para o Brasil. Para as tribos de Discocephalinae e Pentatominae, os números no Brasil estão sendo compilados.

PENTATOMOIDEA - 202/+776'

Acanthosomatidae - 1/1

Hellica nitida Haglund, 1868

Canopidae - 2/6

Canopus burmeisteri McAtee \& Malloch, 1928

Canopus orbicularis Horvath, 1919

Cydnidae - 14/47

Atarsocoris giselleae (Carvalho,1952)

Cyrtomenus (Cyrtomenus) bergi Froeschner, 1960

Cyrtomenus (Cyrtomenus) mirabilis (Perty, 1830)

Cyrtomenus (Syllobus) emarginatus Stål, 1862

Cyrtomenus (Syllobus) teter (Spinola, 1837)

Dallasiellus (Dallasiellus) levipennis (Signoret, 1883)

Dallasiellus (Dallasiellus) lugubris (Stål, 1860)

Dallasiellus (Ecarinoceps) sp.

Melanaethus spinolae (Signoret, 1863)

Pangaeus (Pangaeus) aethiopis (Fabricius, 1787)

Prolobodes giganteus (Burmeister, 1835)

Scaptocoris buckupi Becker, 1967

Scaptocoris castanea Perty, 1833

Tominotus inconspicuus Froeschner, 1960

Dinidoridae - 0/5

Megarididae - 0/6

Pentatomidae - 163/+621

Asopinae - 13/46

Alchaeorhyncus grandis (Dallas, 1851)

Heteroscelis robustus Thomas, 1992

Heteroscelis servillei Laporte, 1833

Oplomus catena (Drury, 1782)

Oplomus salamandra (Burmeister, 1835)

Podisus distinctus (Stål, 1860)

Podisus nigrispinus (Dallas, 1851)

Stiretrus decemguttatus (Lepeletier \& Serville, 1828)

Stiretrus erythrocephalus (Lepeletier \& Serville, 1828)

Supputius cincticeps (Stål, 1860)

Supputius typicus Distant, 1889

Tynacantha marginata Dallas, 1851

Tyranocoris rex Thomas, 1982

Cyrtocorinae - 3/09

Ceratozygum horridum (Germar, 1839)

Cyrtocoris egeris Packauskas \& Schaefer, 1998

Cyrtocoris trigonus (Germar, 1839)

DISCOCEPHALINAE - 25/124

Discocephalini - 18/96

Agaclitus australis Becker \& Grazia, 1992

Antiteuchus macraspis (Perty, 1834)

Antiteuchus mixtus (Fabricius, 1787)

Antiteuchus radians Ruckes, 1964 
Antiteuchus tripterus (Fabricius, 1787)

Cataulax eximius (Stål, 1860)

Dinocoris (Dinocoris) corrosus (Herrich-Schäffer, 1844)

Dinocoris (Dinocoris) gibbosus (Fallou, 1889)

Dinocoris (Dinocoris) gibbus (Dallas, 1852)

Dinocoris (Dinocoris) maculatus (Laporte, 1832)

Dinocoris (Praedinocoris) lineatus (Dallas, 1852)

Discocephala marmorea (Laporte, 1832)

Dryptocephala punctata Amyot \& Serville, 1843

Eurystethus deplanatus Becker, 1966

Eurystethus goianensis Becker, 1966

Eurystethus (Eurystethus) ornatus Ruckes, 1966

Eurystethus (Hispidisoma) variegatus Ruckes, 1966

Sympiezorhincus tristis Spinola, 1837

Ochlerini - 7/29

Catulona pensa Rolston, 1992

Catulona lucida Campos \& Grazia, 2004

Colpocarena sp.

Cromata ornata Rolston, 1992

Macropygium reticulare (Fabricius, 1803)

Miopygium cyclopeltoides Breddin, 1904

Schaefferella incisa (Herrich-Schäffer, 1839)

Edessinae - 14/+100

Brachystethus geniculatus (Fabricius, 1787)

Brachystethus vicinus Signoret, 1851

Edessa albomarginata (Stål, 1855)

Edessa cerradensis Silva, Fernandes \& Grazia, 2006

Edessa collaris Dallas, 1851

Edessa coralipes Erichson, 1848

Edessa elaphus Breddin, 1905

Edessa loxdalii Westwood, 1837

Edessa meditabunda (Fabricius, 1784)

Edessa nigropunctata Silva, Fernandes \& Grazia, 2006

Edessa rufodorsata Silva, Fernandes \& Grazia, 2006

Edessa rufomarginata (De Geer, 1773)

Edessa virididorsata Silva, Fernandes \& Grazia, 2006

Lopadusa (Bothrocoris) quinquedentata (Spinola, 1837)

Lopadusa (Lopadusa) augur Stål, 1860

Peromatus notatus (Burmeister, 1835)

Pentatominae - 108/341

Catacanthini - 8/?

Arocera (Arocera) acroleuca (Perty, 1833)

Arocera (Euopta) nigrorubra (Dallas, 1851)

Arocera (Euopta) placens (Walker, 1867)

Arocera (Euopta) spectabilis (Drury, 1782)

Rhyssocephala rufolimbata (Stål, 1872)

Runibia decorata (Dallas, 1851)

Runibia perspicua (Fabricius, 1798)

Vulsirea violacea (Fabricius, 1803)

Carpocorini - 45/?

Agroecus griseus Dallas, 1851

Agroecus scabricornis (Herrich-Schäffer, 1844)

Berecynthus hastator (Fabricius, 1798)

Curatia denticornis Stål, 1865

Dichelops (Diceraeus) furcatus (Fabricius, 1775)

Dichelops (Diceraeus) melacanthus (Dallas, 1851)

Dichelops (Diceraeus) phoenix Grazia, 1978

Dichelops (Dichelops) avilapiresi Grazia, 1978

Dichelops (Dichelops) leucostigmus (Dallas, 1851)

Dichelops (Dichelops) nigrum Bergroth, 1914 
Dichelops (Dichelops) peruanus Grazia, 1978

Dichelops (Dichelops) pradoi Grazia, 1978

Dichelops (Dichelops) punctatus Spinola, 1837

Euschistus (Euschistus) heros (Fabricius, 1798)

Euschistus (Euschistus) taurulus Berg, 1878

Euschistus (Mitripus) convergens (Herrich-Schäffer, 1842)

Euschistus (Lycipta) hansi Grazia, 1987

Euschistus (Lycipta) illotus Stål, 1860

Euschistus (Lycipta) machadus Rolston, 1982

Euschistus (Lycipta) picticornis Stål, 1872

Euschistus (Lycipta) sharpi Bergroth, 1891

Euschistus (Lycipta) triangulator (Herrich-Schäffer, 1842)

Galedanta bituberculata Amyot \& Serville, 1843

Galedanta compastoides Breddin, 1906

Galedanta rotundicornis Grazia, 1981

Glyphepomis spinosa Campos \& Grazia. 1998

Hypatropis australis Fernandes \& Grazia, 1996

Hypatropis inermis (Stål, 1872)

Ladeaschistus sp.

Mormidea cornicollis Stål, 1860

Mormidea hamulata Stål, 1860

Mormidea maculata Dallas, 1851

Mormidea notulifera Stål, 1860

Mormidea v-luteum (Lichtenstein, 1796)

Mormidea ypsilon (Linnaeus, 1758)

Oebalus poecilus (Dallas, 1851)

Oebalus ypsilongriseus (DeGeer, 1773)

Parahypatropis sinuatus (Stål, 1872)

Paramecocephala subsolana Frey-da-Silva \& Grazia, 2002

Paratibraca infuscata Campos \& Grazia, 1995

Poriptus luctans Stål, 1861

Proxys albopunctulatus (Palisot de Beauvois, 1805)

Proxys victor (Fabricius, 1775)

Sibaria armata (Dallas, 1851)

Tibraca limbativentris Stål, 1860

Menidini $-1 /$ ?

Rio acervatus Fortes \& Grazia, 2000

Nezarini - 12/?

Chinavia brasicola (Rolston, 1983)

Chinavia difficilis (Stål, 1860),

Chinavia erythrocnemis (Berg, 1878)

Chinavia impicticornis (Stål, 1862)

Chinavia longicorialis (Breddin, 1901)

Chinavia nigrodorsata (Breddin, 1901)

Chinavia obstinata (Stål, 1860)

Chinavia pontagrossensis (Frey-da-Silva \& Grazia, 2001)

Chinavia runaspis (Dallas, 1851)

Chinavia ubica (Rolston, 1983)

Nezara viridula (Linnaeus, 1758)

Roferta marginalis (Herrich-Schäffer, 1836)

Pentatomini - 32/?

Arvelius albopunctatus (De Geer, 1773)

Arvelius diluticornis Breddin, 1909

Arvelius paralongirostris Brailovski, 1981

Banasa alboapicata (Stål, 1860)

Banasa angulobata Thomas, 1990

Banasa dubia Thomas, 1990

Banasa lanceolata Thomas, 1990

Banasa nigrifrons Thomas, 1990

Banasa sulcata Thomas, 1990

Chlorocoris (Chlorocoris) complanatus (Guérin-Méneville, 1831)

Chlorocoris (Chlorocoris) tau Spinola, 1837

Evoplitus humeralis (Westwood, 1837) 
Loxa deducta Walker, 1867

Loxa virescens Amyot \& Serville, 1843

Loxa viridis (Palisot de Beauvois, 1805)

Marghita similima Grazia \& Koehler, 1983

Mayrinia curvidens (Mayr, 1864)

Myota aerea (Herrich-Schäffer, 1842)

Pallantia macula (Dallas, 1851)

Pellaea stictica (Dallas, 1851)

Serdia apicicornis Stål, 1860

Serdia concolor Ruckes, 1958

Serdia delphis Thomas \& Rolston, 1985

Serdia indistincta Fortes \& Grazia, 2005

Serdia inspercipes Stål, 1860

Serdia lobata Fortes \& Grazia, 2005

Serdia maxima Fortes \& Grazia, 2005

Serdia robusta Fortes \& Grazia, 2005

Serdia rotundicornis Becker, 1967

Stictochilus tripunctatus Bergroth, 1918

Taurocerus edessoides (Spinola, 1837)

Tibilis apposita Barcellos \& Grazia, 1993

Piezodorini - $1 /$ ?

Piezodorus guildinii (Westwood, 1837)

Procleticini $-1 /$ ?

Thoreyella brasiliensis Spinola, 1850

Pentatominae, sem classificação em nenhuma das tribos

Thyanta (Argosoma) brasiliensis Jensen-Haarup, 1928

Thyanta (Argosoma) humilis Bergroth, 1891

Thyanta (Phacidium) acuta Ruckes, 1952

Thyanta (Phacidium) fimbriata Rider, 1991

Thyanta (Phacidium) robusta Rider, 1991

Thyanta (Thyanta) perditor (Fabricius, 1794)

Phloeidae - 3/3

Phloea corticata (Drury, 1773)

Phloea subquadrata Spinola, 1837

Phloeophana longirostris (Spinola, 1837)

Scutelleridae - 5/+50

Agonosoma flavolineata Laporte, 1832

Orsilochides leucoptera (Germar, 1839)

Coptochilus sp.

Galeacius martini Schouteden, 1904 (Est. Exp. Itirapina - ZUEC)

Pachycoris torridus (Scopoli, 1772)

Tessaratomidae $-1 / 2$

Piezosternum thunbergi Stål, 1860

Thyreocoridae - 13/84

Alkindus crassicosta Horvath, 1919

Corimelaena (Parapora) parana McAtee \& Malloch, 1933

Galgupha (Acrotmetus) schulzii (Fabricius, 1781)

Galgupha (Euryscytus) basalis (Germar, 1839)

Galgupha (Euryscytus) contracta McAtee \& Malloch, 1933

Galgupha (Euryscytus) dificillis (Breddin, 1914)

Galgupha (Euryscytus) rasilis (Horvath, 1919)

Galgupha (Gyrocnemis) concava McAtee \& Malloch, 1933

Galgupha (Gyrocnemis) cruralis (Stål, 1862)

Galgupha (Gyrocnemis) impressa (Horvath, 1919)

Galgupha (Gyrocnemis) triconcava McAtee \& Malloch, 1933

Galgupha (Nothocoris) coccinelloides Horvath, 1919

Galgupha (Nothocoris) terminalis (Walker, 1867) 